\title{
MATHEMATICAL PROBLEMS IN OPERATIONS RESEARCH
}

\author{
PHILIP M. MORSE
}

During the past war a new field of application of the scientific method was developed, called operations research, or operational analysis. A number of mathematicians contributed to this development and some of their work should be of general interest to mathematicians, though reasons of military security have precluded publication of much of it. It is my intention to discuss a number of problems in this field which can now be talked about, and to indicate why further work in operations research is of some importance at present.

The term operations research has heretofore been used to connote various attempts to study the operations of war by scientific methods. From a more general viewpoint, however, operations research can be considered to be an attempt to study those operations of modern society which involve organizations of men or of men and machines. The study is behavioristic, not subjective, and the aim is to be able to predict the changes produced in the operation by changes in external conditions. The subject can be said to cover, for example, the quantitative aspects of city planning (including traffic analysis), those parts of the study of telephone traffic handling which are called systems engineering, and certain aspects of efficiency engineering. Its methods might be applied to a scientific study of peacetime operations, such as those of railroads or of other transportation systems, if this were desired. In each case one seeks to study the dependence of certain measures of effectiveness of an operation upon certain operational parameters which are subject to the control of the director of the operation. It is important to appreciate what operations research is not, as well as what it is: it is not efficiency engineering or historical analysis nor is it a branch of applied statistics. It views operations from a much more active and experimental point of view than do these other subjects.

It is obvious that operations research is a branch of engineering, rather than of pure science, since it is concerned with the application of scientific method to immediate and pressing practical problems. At least in the initial stages it must be developed in close connection with these practical problems and in personal contact with the administrators who have control over the operation under study. The

The twenty-first Josiah Willard Gibbs lecture delivered at Athens, Georgia, December 29, 1947, under the auspices of the American Mathematical Society; received by the editors January $9,1948$. 
fundamental data concerning the operation must be obtained in part by first-hand observation and in part by judicious variation of the operational parameters involved. After the data are collected the results of their analysis are of value to the administrator in charge, in helping him plan future operations.

As with any field of engineering, a number of branches of science are called upon for help. For example, physics is required for a basic understanding of the operational possibilities of the machines involved, and biophysics and psychophysics are required in order to understand the capabilities of the human components. Mathematics is (as always) basic to all of the work, and some of the problems already encountered have required mathematical abilities of fairly high order to solve. During the war it was found that a mathematician, a physicist and a biologist together make up a research team of considerable effectiveness. The biologist is often useful chiefly for his ability to draw mechanistic inferences from a little data concerning very complex systems.

Operations research is still of considerable importance, and there are at present more positions open in the field than there are compentent workers to fill them. Because of the success of several operations research groups during the war the armed services of this country all wish to maintain groups of this sort now; and in the present state of international relations, it is probably important that such groups be maintained. In addition, a number of large industries have shown interest in applying the same techniques to improving their own operations.

The writer wishes to call the attention of mathematicians to these opportunities, and to indicate, by a group of examples, that problems of mathematical interest are encountered in operations research. The group of problems chosen for discussion here are from the military field, because up to the present, the military applications have been most intensively studied. The examples were chosen for their mathematical interest, not necessarily for their practical importance. Some of the results could have peace-time application, of course; this will be seen as the discussion progresses. Further work in peacetime operations research will certainly bring to light other problems of equal or perhaps greater mathematical interest.

To sum up this preliminary discussion in concise form:

1. Operations research (in its military application) is the quantitative study of a strategic or a tactical operation, or a part of one.

2. It gets its data from combat or trial action reports and from physical or psychological tests of the mean and machines involved. 
3. It uses idealized and simplified pictures of the operation (as all branches of applied mathematics do) to serve as the basis of analytic calculation and prediction.

4. It has an amply demonstrated practical value, and it is capable of wide application (cf. mathematical economics, traffic analysis, epidemiology, systems engineering, and so on).

5. It can lead to new and challenging problems in mathematics, as some of the following discussion may indicate.

The search problem. A very large class of problems encountered in naval and air operations is related to the process of search; an observer, equipped with some means of making contact with a target, moves or is moved over an area or volume in some more or less regular pattern of search; the problem is to find the pattern which most efficiently results in contact under specified circumstances. The problem is applicable to many cases: the means of contact may be visual, by radar or sonar; the means of transport of the observer may be by aircraft or beneath the water; the "observer" may be a proximity-fused guided missile, and the "contact" desired may be the destruction of the target; peacetime applications to geological prospecting are obvious, and so on. Mathematically, the problem reduces often to one of calculus of variations, but, in many cases, with "something new added."

The problem ${ }^{1}$ usually can be divided into a number of parts: the contact problem, dealing with the relationship between the physical properties of the detection equipment and the probability of contact with the target, when observer and target are in given relative positions; the track or pattern problem, dealing with the determination of the optimum pattern of search for given conditions; and the tactical problem, dealing with the reciprocal effects occurring when the target is also provided with detection equipment. Analyses of the tactical problem often require the techniques ${ }^{2}$ developed by von Neumann for his theory of games. All aspects of the search problem involve fundamental concepts and techniques of the theory of probability, expressed from a point of view enough different from that of classical

${ }^{1}$ Much of the discussion here follows the work of B. O. Koopman, who made major contributions to this field while working as a member of the Operations Research Group (now Operations Evaluation Group) U. S. Navy Office of the Chief of Naval Operations.

${ }^{2}$ Theory of games and economic behaviour by J. von Neumann and O. Morgenstern, Princeton University Press, 1944. 
probability theory as to cast new light on some of its concepts and techniques.

Probability of contact. The mathematical aspects of the contact problem start with the definition of the instantaneous probability of contact, $g$. Nearly all detection equipment searches by means of "glimpses" : a radar set sends out a sequence of pulses, which may or may not return a measurable echo from the target; a sonar set behaves similarly, though with longer intervals between pulses; even the human eye searches the horizon in a series of momentary "fixations." The probability contours for single-glimpse detection of an object on the surface of the ocean by eye or by radar are plotted in

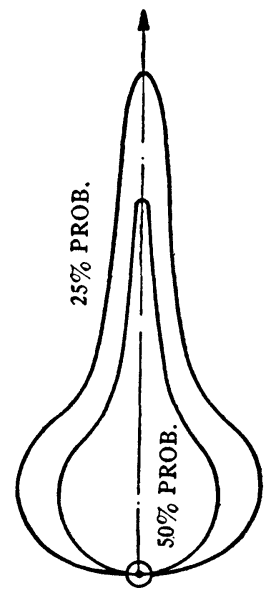

VISUAL GLIMPSE

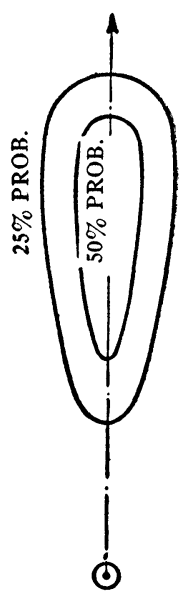

RADAR PULSE

FIG. 1. Polar contours of instantaneous probability of detecting target on ocean surface for single eye fixation and for single radar pulse. Observer is at $O$.

polar diagrams in Fig. 1, as functions of range and horizontal angle. By way of contrast, the contours for probability of "contact" (that is, of destruction) for a single bomb would be concentric circles, the probability $g$ here being simply a function of $r$, the distance between the target and the point of impact of the bomb.

These instantaneous probabilities are next combined to obtain the probability of detection (or destruction) by any of a number of glimpses (or bombs). It turns out that the individual probabilities are independent of each other in most of the cases studied, so the usual rules for combination of probabilities can be applied. The probability that a contact is made in at least one of $n$ glimpses is 


$$
\begin{aligned}
P & =1-\left(1-g_{1}\right)\left(1-g_{2}\right) \cdots\left(1-g_{n}\right) \\
& =1-\exp \left[-\sum_{m=1}^{n} \Phi_{m}\right] ; \quad \Phi_{m}=-\ln \left(1-g_{m}\right) .
\end{aligned}
$$

As long as the glimpse probabilities are independent the quantities $\Phi_{m}$ are additive; for this reason they are called contact potentials.

This equation suffices for the basis of a large number of problems connected with gunnery and area bombardment. Combination of probabilities of destruction with probabilities representing gunnery or bombing errors will enable over-all probabilities to be computed and optimum patterns for different targets to be determined. In some cases of practical importance (as in rapid-fire gunnery) successive probabilities are not independent, and the above equation is not valid.

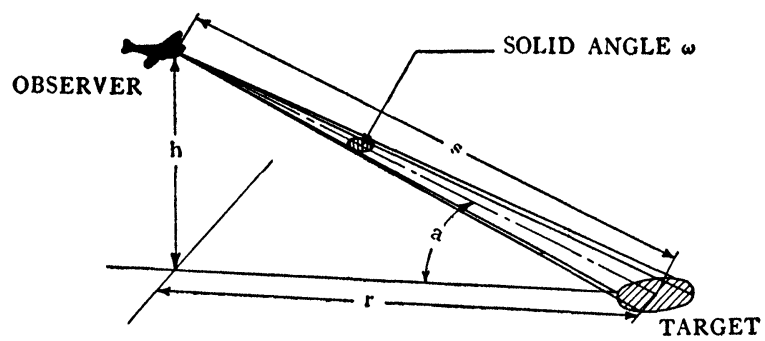

FIG. 2. Visual detection of wake on ocean surface by observer in aircraft.

In the case of actual search for a target (on the surface of the ocean, for instance) we must take into account the fact that the search goes on continuously as the observer moves about. During a time $d t$ which is short compared to the total time of search but which is long compared to the average time between glimpses, the eye or radar set scans the horizon about equally well in all directions. Therefore the probability of making contact within the time interval $d t$ can be written as $\gamma(r) d t$, where the quantity $\gamma$ is usually independent of the angle between the line of sight and the direction of motion of observer or target. The quantity $\gamma$ depends on $r$, the horizontal distance between observer and target, and on the visibility conditions (atmospheric haziness, state of repair of radar set, and so on) at the time of search.

For instance, within rather narrow limits, the probability rate $\gamma$ for visual detection of a distant object is proportional to the solid angle subtended by the object at the eye. If the object searched for is a wake of constant area on the surface of the ocean and if the observer 
is in an aircraft at height $h$ and horizontal range $r$ from the target, study of Fig. 2 will show that if $r \gg h$,

$$
\boldsymbol{\gamma}=\kappa \omega=\left(B / s^{2}\right) \sin \alpha=\left(B h / s^{3}\right) \simeq\left(B h / r^{3}\right),
$$

where the quantity $B$ depends on the area and contrast of the wake, the amount of haze in the air, and so on. This is known as the inverse cube law for contact rate. This equation is only valid for search for small objects in conditions of good visibility, but it serves as a good example, which can be handled analytically.

Lateral range and search width. Next we must consider the effect of the motion of the observer with respect to the target. As shown in

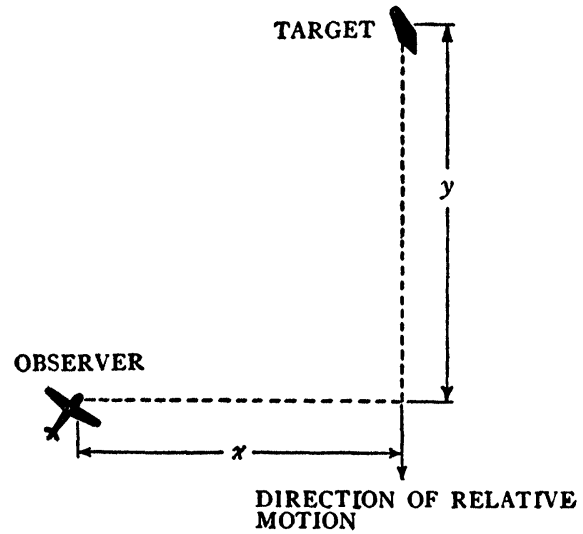

FIG. 3. Relative motion of target past observer, relative velocity is $v$ and lateral range is $x$. Probability of contact during transit.

Fig. 3, we picture this most easily by considering the observer to be at rest and we assume that the relative velocity is constant both in magnitude, $v$, and in direction, as long as the two are within detection range. For a certain initial configuration the distance of closest approach will be $x$ (which is called the lateral range). The position of the target along its path of relative motion is given by $y$.

It should be obvious that the probability of contact during the time while the observer is passing by the target is given by the formula

$$
p(x)=1-\exp \left[-\frac{1}{v} \int_{-\infty}^{\infty} \gamma\left(\left(x^{2}+y^{2}\right)^{1 / 2}\right) d y\right]
$$


For instance, for the inverse cube law given in Equation 2, the contact probability as function of lateral range turns out to be

$$
p(x)=1-e^{-2 \beta / x^{2}} ; \quad \beta=(B h / v) .
$$

Possible curves for contact probability $\gamma(\boldsymbol{r})$ and corresponding lateral range probability are shown in Fig. 4 . In the first case, the probability of contact increases so rapidly at range $R$ that there is almost certainty of detection whenever the lateral range is less than $R$. This is called the definite range case. The other case shown is that for the inverse cube law.

(a)
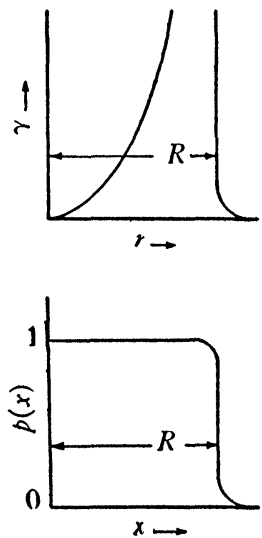

(b)
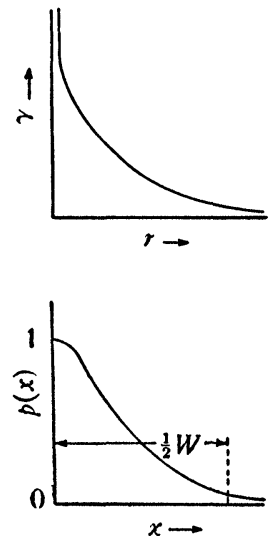

FIg. 4. Curves for instantaneous contact rate $\gamma(r)$ together with the corresponding lateral range probability, $p(x)$.

Case (a) is the definite range case.

Case (b) is for the inverse cube law.

Now suppose that the target is at rest somewhere along a line of length $D$ (long compared to the range of detection) at right angles to the motion of the observer. If the observer's track crosses this line at some randomly chosen point, independent of the position of the target, then the probability that contact will be achieved turns out to be

$$
P=\frac{W}{D} ; \quad W=\int_{-\infty}^{\infty} p(x) d x
$$


where $W$ is called the effective search width for the particular observertarget pair under study. For the definite range case, $W=2 R$, where $R$ is the definite range. For the inverse cube law,

$$
W=2(2 \pi \beta)^{1 / 2}=2(2 \pi(B h / v))^{1 / 2} .
$$

As might be expected, the search width is a useful measure of the effectiveness of a piece of detection equipment. The quantity $v W$, called the search rate, gives the effective number of square miles which can be searched over in an hour (if $W$ is in miles and $v$ in miles per hr.). For the inverse cube law the search rate is proportional to the square root of the velocity and of the altitude of the plane (assuming a cloudless day). In actual practice the dependence of search rate on velocity is not as simple as this example would indicate.

Search pattern. A problem of some interest, and one which is basic to the question of search pattern mentioned earlier, is: a single target is placed at random somewhere inside an area $A$, whose dimensions are large compared to the search width $W$; what is the probability that, after $T$ hours of search by the observer, the target is found? The answer will, of course, depend on how the search is carried out, that is, on the pattern of search. We shall obtain the answer for two different sorts of search pattern.

As one extreme, we assume that the search is completely random; the track of the observer (or observers, for several identical observers may take part) consists of pieces of straight line, of various lengths, placed at random within the area $A$, and covering it in a more or less uniform manner. For this search pattern the probability that the target be passed at a lateral range $x$ is independent of $x$, and the chance of finding the target in time $d T$ is just the search rate, $v W$, times $d T$, divided by $A$. Therefore the probability of making contact at some time during the search is

$$
P=1-e^{-W L / A}
$$

where $L=v T$ is the total length of track passed over by the observer (or observers) during the search.

Another search pattern, somewhat more efficient, is to cover area $A$ with straight, parallel tracks in as uniform a manner as possible. If the total track length is to be $L$, the spacing between parallel tracks is $S=(A / L)$. If the target is a lateral distance $x$ from one of the tracks it can be shown that the probability of contact is 


$$
\begin{aligned}
P(x, S) & =1-e^{-\Phi(x, S)} \\
\Phi(x, S) & =-\sum_{n=-\infty}^{\infty} \ln [1-p(x-n S)] \\
& =\prod_{n=-\infty}^{\infty} \frac{1}{v} \int_{-\infty}^{\infty} \gamma\left(\left(y^{2}+(x-n S)^{2}\right)^{1 / 2}\right) d y
\end{aligned}
$$

where $p(x)$ is the lateral range probability defined in Equation 3.

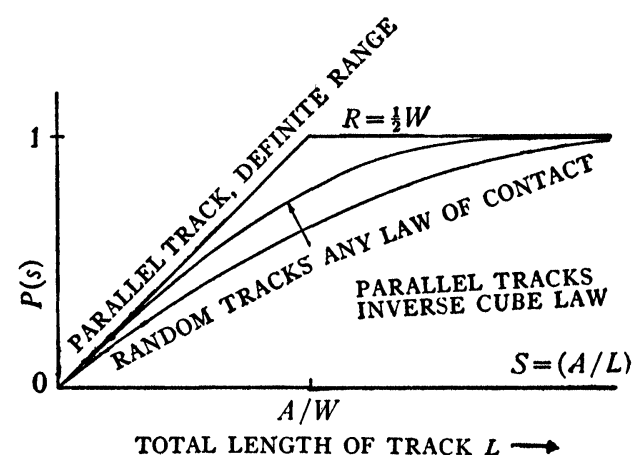

Frg. 5. Probability of contact during search of track length $L$, for single target at random in area $A$.

Since the target is placed at random in the area the probability of contact is the average value of $P$ :

$$
P(S)=\frac{1}{S} \int_{0}^{S} P(x, S) d x .
$$

For the definite range case, where $p(x)$ is zero for $x>R$ (where $2 R=W)$ and unity for $x<R, P(S)$ is equal to $(2 R / S)=(W L / A)$ whenever $S$ is larger than $2 R$ (in this case the searched-over paths do not completely cover the area); and is equal to unity when $S$ is smaller than $2 R$ (in this case the area searched covers $A$ completely). For the inverse cube law, discussed earlier, the result is

$$
P(S)=\operatorname{erf}\left(\pi(2 \beta)^{1 / 2} / S\right)=\operatorname{erf}\left(\pi^{1 / 2} W / 2 S\right) .
$$

Inspection of Fig. 5, which shows the contact probability as function of total track length $L$ for the three cases discussed, displays the amount of advantage to be gained by care in arranging the pattern of search. Purely random search introduces inevitably a certain amount of overlapping of search effort, which is eliminated by the use 
of parallel, equidistant tracks. For the definite range case, no overlap occurs until the track spacing $S$ becomes smaller than $2 R$. The inverse cube law case represents an intermediate case, in that a certain amount of overlap of search effort occurs even with parallel tracks. In actual practice it is quite difficult to follow exactly parallel, exactly equidistant search tracks, and careful navigation is required to obtain search efficiencies appreciably larger than the efficiency corresponding to the curve for random search.

Of course it is easy to design a search pattern which would be considerably less effective than the curve for purely random search. Concentration of search effort in one part of the area, for instance, will reduce the probability (as long as the target is equally likely to be anywhere). On the whole, however, any search effort which attempts to cover the area in a reasonably uniform manner will produce results approximately equal to the random search case, and considerable care in navigation will enable markedly better results to be attained. In the succeeding parts of this paper the formula for probability of contact for random search, given in Equation 6, will be used as a minimum attainable measure of effectiveness; any reasonably well designed and executed search pattern should do better than this, none should do worse unless they are badly designed.

We next consider a somewhat more complicated case, where the target may be in one or the other of two areas, $A_{1}$ and $A_{2}$. The probability that it is in $A_{1}$ is $p_{1}$, with its whereabouts within the area unknown; the probability that it is in $A_{2}$ is $p_{2}=1-p_{1}$. The problem is as follows: suppose we have a certain amount of searching effort, represented by the track length $L$; how do we distribute this effort between $A_{1}$ and $A_{2}$ to have the best chance of finding the target?

If we try assigning a length of track $x$ to area $A_{1}$ and distribute it uniformly over the area (so we can use Equation 6 for the chance of contact), then a track-length $(L-x)$ will be left to be used in area $A_{2}$ and the contact probability will be

$$
P(x)=1-p_{1} e^{-\left(W / A_{1}\right) x}-p_{2} e^{-\left(W / A_{2}\right)(L-x)} .
$$

We have therefore to make $P(x)$ a maximum, or $p_{1} e^{-\left(W / A_{1}\right) x}$ $+p_{2} e^{-\left(W / A_{2}\right)(L-x)}$ a minimum, in the range $0 \leqq x \leqq L$.

To make the discussion more concrete, let us assume that the probability density $\left(p_{1} / A_{1}\right)$ for the target in the first area is greater than that in the second area. We start with a small allowed track length $L$ and investigate how the best distribution of search effort changes as we have more and more track length available. For a small enough value of $L$, we have $\left(p_{2} / A_{2}\right) \leqq\left(p_{1} / A_{1}\right) e^{-\left(W L / A_{2}\right)}$. When this is true the 
quantity $p_{1} e^{-\left(W / A_{1}\right) x}+p_{2} e^{-\left(W / A_{2}\right)(1-x)}$ has no minimum for $x$ in the range $0<x<L$, its least value is for $x=L$ corresponding to all search in $A_{1}$. In other words, if the available search effort is small enough we should concentrate this effort in the most promising area and disregard the other area entirely. This is a result which is not of ten encountered in classical calculus of variations problems, but which of ten turns up in operations research problems.

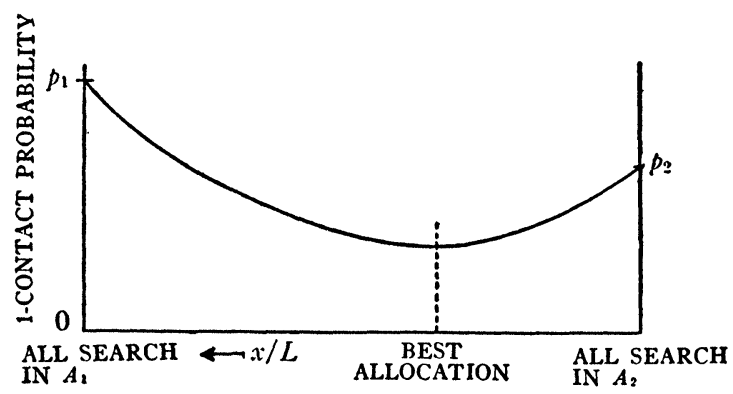

Fig. 6. Calculation of optimum distribution of search effort between two areas $A_{1}$ and $A_{2}$ with $p_{1}$ the probability the target is in $A_{1}$ and $p_{2}$ the probability it is in $A_{2}$. Total track length $L$, track length in $A_{1}$ is $x$. Probability of contact for random search.

If now the available path length $L$ is sufficiently increased there will be a maximum for $P(x)$ in the range $0<x<L$ as shown in Fig. 6, and the usual process of maximization will obtain the proper answer for $x$. It is of interest to note that, if $L$ is large enough, the solution indicates that more time should be spent in $A_{2}$ than in $A_{1}$, although $A_{2}$ is to be ignored when $L$ is small. If there is plenty of effort available it is worthwhile to spend a lot of it on the less rewarding area.

These very simple examples will perhaps show how the searchpattern problem can be attacked, and may perhaps indicate the difficulties which are encountered as soon as less simple cases are tackled. The difficulties, it should be brought out, are mathematical ones and not ones of boring detail. The solution of a search-pattern problem which includes, for instance, a certain distribution in velocity on the part of the target requires mathematical skill of no mean order. For that matter, the comparatively simple problem of determining the optimum pattern of search track when the probability density of the target varies from point to point is beyond the present capacities of the calculus of variations. The integral giving the probability of detection can be written down for each chosen pattern of track; but 
if one attempts to compute the optimum pattern, an integral equation is obtained which so far defies even formal solution.

And yet these more complex problems should be solved. We have spoken here only of two-dimensional search problems, typical of a surface fleet. But if "push-button warfare" should become a reality, three-dimensional search will be a matter of life or death, and the time allowed to find and destroy a guided missile headed for our shores is likely to be limited indeed.

A point of interest in theory of probability can be mentioned here. In practice one needs the value of the effective search width $W$ before one can lay out search patterns. This value is obtained from data collected from past operations. It is, of course, an average value, averaged over the fluctuations of $W$ which occur in normal operation. These fluctuations are of two types: the short-term fluctuations which occur during an individual search, due to rolling and pitching of the target, momentary fluctuations in attention of the observer, and so on; and long-term fluctuations, which occur from trip to trip, due to changes in atmospheric properties or instrument maintenance, etc. The value of $W$ obtained from past operational data is usually an average over both long and short-term fluctuations. But is this the best value of $W$ to use in laying out the next individual search? If the atmosphere or equipment is in top form one day is there some way of finding this out and/or taking advantage of it? Here is a point which strikes close to the fundamentals of probability theory.

Measure and countermeasure. In operations research one often encounters the following sort of problem $:^{3}$ if $A$ uses one tactic, $B$ should use a certain defense, but if $A$ uses another tactic, $B$ should do something else; what are the best tactics for both $A$ and $B$ ? Problems of this sort are discussed by von Neumann and Morgenstern ${ }^{2}$ in their Theory of games. In some of the problems they discuss the answer is definite; there is one particular action which $A$ must take, and likewise for $B$, in order that neither $A$ nor $B$ shall lose too much. In other words, if $A$ took any other action than the recommended one, $B$ could take advantage of him, and vice versa.

In other problems the answer is indefinite; any one action on the part of $A$ will eventually lead to trouble if the "game" is played several times; what is needed is a variation of tactics, in a random

${ }^{3}$ The following examples are taken from some of the work done by the author and G. E. Kimball while they were with the Operations Research Group, U.S.N. 
manner, so that $B$ is never sure which action $A$ will take next time. This sort of situation often turns up in operations research. The enemy may have a countermeasure to some weapon of ours which renders the weapon a liability in certain cases. Should we use the weapon or not?

An example of this type of analysis can again be taken from the theory of search. A submarine wishes to pass through a channel without being detected by a patrol aircraft, which flies back and forth
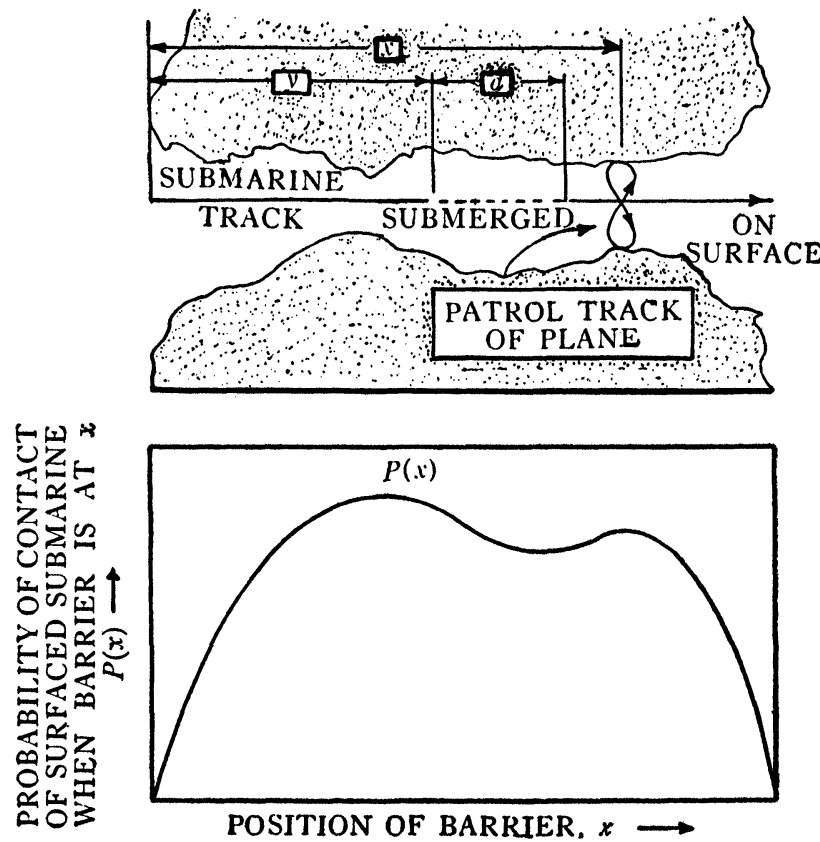

FIg. 7. Barrier patrol versus diving submarine. $P(x)=$ Probability of contact if barrier is at $x$ and submarine is surfaced.

across the channel each day. The submarine can escape detection by submerging, but we assume that it can only travel a total distance $a$ submerged and that $a$ is shorter than the channel length. It can surface and dive again several times, but the total length of path submerged is no larger than $a$.

The channel is supposed to be wide enough so that only a few trips are possible by the plane each day. Consequently each day the plane must choose which point along the channel it is to patrol across. If it chooses a wide part the number of traverses will be fewer and the chance of detection, even if the submarine is surfaced at that point, 
is less than if the plane were patrolling across a narrower part. In fact, one can plot a curve giving the probability $P(x)$ that the plane will discover the submarine if it patrols across the portion a distance $x$ from one end of the channel and if the submarine is not submerged when passing this part. Such a curve is given in Fig. 7.

It might seem, at first sight, that the aircraft should always patrol where $\boldsymbol{P}$ is largest. But if this action were taken the submarine would soon learn to dive when it goes past that point and the plane would never see the submarine. Obviously the plane must spread out its effort, one day patrol at one value of $x$, next day at another and so on, with the frequency of patrol at $x$ governed by some predetermined function $\phi(x)$, which is called the density of the barrier at $x$. This patrol effort should not be distributed from point to point in a regular manner, or the opponent could take advantage of this; the assignment of patrols should be in a random manner, subject to the density function $\phi(x)$.

Similarly, if the submarine is always submerged in one region the plane could patrol outside that region and the submarine would lose the advantage of its submergence. It must vary its regions of submergence in a random manner, with a relative frequency according to a density function $\psi(x)$. To recapitulate, equation-wise:

$$
\begin{aligned}
& \text { Density of patrol }=\phi(x) ; \quad \int \phi(x) d x=1, \\
& \text { Prob. of submergence }=\psi(x) ; \quad \int \psi(x) d x=a,
\end{aligned}
$$$$
\text { Prob. of detection } G=\int P(x) \phi(x)[1-\psi(x)] d x \text {. }
$$

First let us consider the way in which the submarine commander must analyze the situation to decide on the submergence density $\psi(x)$. He must choose $\psi$ so that if the opposing side learned what $\psi$ he was using they could not gain an advantage, but if he learned the patrol plans of the plane (the values of $\phi$ ) he might be able to gain an advantage.

If the airplane patrol-squadron commander found out that the submergence density function for the submarine was to be $\psi(x)$, he could make the probability of contact $G$ a maximum by flying his patrol plane at the point $x$ where $P(x)[1-\psi(x)]$ has its maximum value. The submarine commander would, of course, like to make the quantity $P(x)[1-\psi(x)]$ equal to zero, everywhere, but this is not 
possible, since the channel is longer than the maximum range of submergence, $a$. The next best thing for the submarine commander to do is to arrange so that $P(x)[1-\psi(x)]$ has no single maximum, that is, is flat along the top. This is done as follows:

Safe tactics for submarine. Over as great a range of $x$ as possible, choose $\psi(x)$ so that

$$
\begin{aligned}
& P(x)[1-\psi(x)]=H, \quad \text { a constant, that is } \\
& \psi(x)=\left\{\begin{array}{cl}
1-\frac{H}{P(x)}, & \text { when } P(x)>H \text { (over range } B \text { of } x), \\
0, & \text { when } P(x)<H \text { (outside range } B \text { of } x \text { ). }
\end{array}\right.
\end{aligned}
$$

The value of $H$ is determined by the submergence limitations of the submarine,

$$
\int \psi d x=\int_{B}\left(1-\frac{H}{P(x)}\right) d x=a, \begin{aligned}
& \text { where the integration is only over } \\
& \text { range } B \text { of } x .
\end{aligned}
$$

If the submarine follows these submergence tactics then no matter what tactics the patrol plane follows, the probability of contact will never be greater than $H$, and it may well be less than $H$ if the patrol plan is badly placed. The integral determining $H$ ensures that $H$ is as small as possible, consistent with the submergence capabilities of the submarine.

These are the safe tactics for the submarine. If the submarine commander learns the plans of the aircraft patrol, he can alter $\psi$ so that $G$, the contact probability, is less than $H$; but if he is not sure about the air patrol plans then a choice of the tactics of Equation 11 will ensure that the probability of contact is never larger than $H$ even if the opposing side has a perfect spy system.

The details of the solution are shown in the left-hand side of Fig. 8. We plot the reciprocal of $P(x)$ and draw a horizontal line of height $(1 / H)$ so adjusted that the area between this line and the curve $(1 / P)$ is equal to $(a / H)$. The difference between this line and the $(1 / P)$ curve, multiplied by $H$, will then be $\psi$ which is plotted in the lower left-hand figure. If the air patrol is placed anywhere between $x_{0}$ and $x_{1}$ the probability of contact $G$ is equal to $H$ : if it is placed outside this range $G$ will be less than $H$.

We note that if $a$ is small enough, or if the value of $P(x)$ fluctuates more widely, the line at height $(1 / H)$ may cut the line $(1 / P)$ at more than two points. When this is the case the region for submergence is divided into two or more parts; those regions for which $(1 / P)$ is larger 
than $(1 / H)$ are excluded from the diving schedule $(\psi=0$ there) and for those regions where $(1 / P)$ is smaller than $(1 / H), \psi$ will equal $1-(H / P)$ as before. This illustrates again a property of many solutions of operational problems: if the resources available are limited they should be used only in the more promising regions, the less promising regions should be disregarded entirely. The same result turned up in the example, quoted earlier, of the search of two areas. It turns up in many problems discussed by von Neumann and Morgenstern. ${ }^{2}$

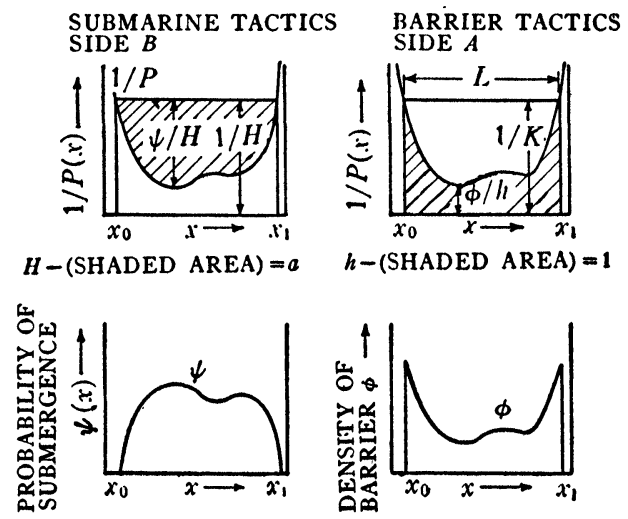

Fig. 8. Solutions of problem shown in figure 7 for safe tactics for both sides.

This property of solutions of operational problems should not be surprising to us (though it is not a usual result in the classical examples of the calculus of variations) for many of these problems are minimax problems with definite boundaries imposed on the parameters by reason of force limitations. Hence it of ten happens that the minimax is on one of the boundaries (where one of the parameters is zero, for instance) instead of inside the boundary.

Now let us see what the patrol squadron commander must do to ensure that the contact probability $G$ be as large as possible. We assume that he knows that the submarine can only travel a distance $a$ under water. If he knew, in addition, that the submarine was submerging according to the schedule $\psi$ he could fly his patrol anywhere between $x_{0}$ and $x_{1}$ and get the same value of $G$. But flying the patrol always at one point (or even at several points) is a dangerous thing to do, for if the submarine commander were to learn this he could alter $\psi$ so as to reduce $G$. The aircraft commander must fly his patrol at random along $x$, with a frequency predetermined by a 
probability density factor $\phi(x)$ (see Equation 10), such that if the submarine commander found out the shape of $\phi$ he would not gain any advantage (he could not lower $G$ ) but if the aircraft commander found out the submarine's schedules, a change of patrol might enable $G$ to be increased. This requirement, we see, is symmetric to that for the submarine. As with the submarine, the barrier density $\phi$ must be adjusted so that the quantity $P(x) \phi(x)$ which comes into the integral for $G$ has no maxima, but is flat on top. Therefore no barrier will be flown for those ranges of $x$ where $P$ is less than some limiting value $K$; and where $P$ is larger than $K$ the barrier density $\phi$ will be inversely proportional to $P$, so that $P \phi$ be a constant in those regions. Therefore we have:

Safe tactics for patrol aircraft. Choose $\phi(x)$ so that $P(\phi)$ equals some constant $h$ over a range of $x$,

$$
\phi(x)=\left\{\begin{array}{cl}
\frac{h}{P(x)}, & \text { when } P(x)>K(\text { over range } C \text { of } x), \\
0, & \text { when } P(x)<K \text { (outside range } C \text { of } x),
\end{array}\right.
$$

where $h$ is chosen so that $\int \phi d x=1$, that is, $(1 / h)=\int_{C}(1 / P) d x$, with the integral taken only over range $C$ of $x$. The length $L$ of the region patrolled is the length of range, $\int_{c} d x=L$.

Assuming that the submarine submerges within range $C$ of $x$, the probability of contact $G$ would be $h(L-a)$; in any case $G$ would never be less than $h(L-a)$ for the patrol schedule $\phi$ given in Equation 12. We have not yet chosen the value of $K$, and therefore the length $L$ of range $C$ of $x$. It certainly would not be advisable to make $L$ smaller than $a$, for then the submarine could dive under the whole barrier. On the other hand it would not do to make $L$ too large, for then $h$ would become too small. In fact, we must adjust $L$ and $h$ so that $h(L-a)$ is a maximum.

A certain amount of algebraic manipulation is required to show that the requirement for $h(L-a)$ to be maximum is equivalent to the requirement that $K=H$, or that range $C$ for the aircraft is identical with range $B$ for the submarine submergence. This is of course reasonable, for any lack of coincidence on the part of either side would represent wasted effort.

The graphical method of determining the patrol schedule $\phi(x)$ is shown in the right-hand side of Fig. 8. One plots again the reciprocal of $P(x)$, draws the line of height $(1 / K)=(1 / H)$ and determines the 
range $C(=B)$ of $x$. We choose the normalizing factor $h$, such that $h$ times the shaded area equals unity. Then the best the submarine can do if the aircraft patrol is alert, and the best the aircraft can do if the submarine commander is clever, is the set of tactics outlined in Equations 11 and 12 and in Fig. 8, resulting in the probability of contact $G=H=h(L-a)$. Any departure from these tactics by one side will enable the other side to gain an advantage if the departure is ascertained.

The problem considered here is an extremely simplified example of a large class of problems which turn up continuously in tactical studies and in many peacetime applications of operations research. The difficulties of solving such problems are not ones of tedious detail, but are of ten due to lack of fundamental techniques. Much more basic research must be carried out before many problems of practical importance can be solved.

Lanchester's laws. One final example, from the field of strategy this time, deals with the relation ${ }^{4}$ between size of opposing forces and the casualty rates in combat of various types. Suppose that at time $t$ there are $m$ units of one side engaging $n$ units of the other side. The time rate of decrease of $m$ and $n$ as the battle continues will depend on the nature of the engaging units and the types of weapons used. For some types of engagement the relations are:

$$
\begin{gathered}
\frac{d m}{d t}=-A, \quad \frac{d n}{d t}=-E A, \quad \frac{d n}{d m}=E, \\
n_{0}-n=E\left(m_{0}-m\right)
\end{gathered}
$$

where the constant $E$, called the exchange rate, is a measure of the relative efficiency of the weapons used by the two sides. This relationship is called Lanchester's linear law of combat.

Another type of engagement corresponds to the equations

$$
\begin{gathered}
\frac{d m}{d t}=-\alpha n, \quad \frac{d n}{d t}=-\alpha E m, \quad \frac{d n}{d m}=(m E / n) \\
n_{0}^{2}-n^{2}=E\left(m_{0}^{2}-m^{2}\right) .
\end{gathered}
$$

This relationship is called Lanchester's square law of combat. It is more often applicable to engagements of modern warfare (where gun fire rather than hand-to-hand combat predominates) than is the

${ }^{4}$ See Aircraft in warfare by F. W. Lanchester, London, Constable, 1916, for a discussion of some of the relations referred to here. 
linear law. Both sets of equations are related mathematically to the equations governing biological equilibria, epidemiology and theory of evolution. ${ }^{5}$

The solutions of these and related differential equations have been obtained and the consequences discussed in some detail. ${ }^{4}$ For instance, from the solution of the square law the strategic advantages of the concentration of force are at once apparent, for a doubling of the number of units in any engagement is worth as much as the quadrupling of the weapon effectiveness.

Solutions of the differential equations do not tell the whole story, however, for they give only the results of a sort of "average engagement," and do not indicate the sort of fluctuations which might be expected in this non-regular world. What is needed is a detailed statistical analysis of the problem.

For the linear law this is not difficult. We define a "unit combat" as that portion of the engagement during which, on the average, $1 /(1+E)$ red units ( $m$ 's) are lost and $E /(1+E)$ blue units $(n$ 's) are lost. After $T$ combats the probability that $\alpha$ red units and $\beta$ blue units are actually lost is, of course,

$$
\begin{aligned}
P(\alpha, \beta) & =\frac{T !}{\alpha ! \beta !}=\frac{E^{\alpha}}{(1+E)^{T}} ; \\
T & =\alpha+\beta ; \quad \alpha<m_{0} ; \quad \beta<n_{0} .
\end{aligned}
$$

Special consideration is required to obtain expressions for $P\left(\alpha, n_{0}\right)$, the probability that the engagement is ended with all of the blue units lost and $\left(m_{0}-\alpha\right)$ red units left, and for the symmetrically related $P\left(m_{0}, \beta\right)$ corresponding to annihilation of the red forces. The results are

$$
P\left(\alpha, n_{0}\right)=\frac{\left(\alpha+n_{0}-1\right) !}{\alpha !\left(n_{0}-1\right) !} \frac{E^{\alpha}}{(1+E)^{\alpha+n_{0}}}, \quad P\left(m_{0}, n_{0}\right)=0 .
$$

From these probabilities one can calculate the chances of any deviation from the solution of the differential equation 13.

A similar analysis of the square law is more difficult, for here we cannot eliminate the time from explicitly entering the formulas. The probability function here is $P(m, n, t)$, the probability that at time $t$ there are $m$ red units and $n$ blue units still unharmed. We change the time scale so that during $d t$, the chance that a red unit is hit is

'See, for instance, V. Volterra, The theory of the struggle for life, Gouthiers-Villars, Paris, 1931, and A. V. Lotka, Elements of physical biology, Williams and Wilkins, Baltimore, 1925. 
$\left(n d t / E^{1 / 2}\right)$ and the chance that a blue unit is hit is $\left(m d t E^{1 / 2}\right)$. Then a consideration of the interrelationship of the functions $P$ shows that

$$
\begin{aligned}
\frac{d}{d t} P(m, n, t)= & \left(m E^{1 / 2}\right)[P(m, n+1, t)-P(m, n, t)] \\
& +\left(n / E^{1 / 2}\right)[P(m+1, n, t)-P(m, n, t)], \\
\frac{d}{d t} P(m, 0, t)= & \left(m E^{1 / 2}\right) P(m, 1, t), \\
\frac{d}{d t} P(0, n, t)= & \left(n / E^{1 / 2}\right) P(1, n, t) .
\end{aligned}
$$

This set of equations should be solved subject to the initial condition that $P\left(m_{0}, n_{0}, t\right)$ is unity and all other $P$ 's are zero at $t=0$.

Investigation of these and similar equations for other types of combat have not received the detailed study they merit. Various approximate and asymptotic solutions of Equations 15 and 17 are needed in order to show general trends more clearly than can the very complicated exact solutions. Other equations, corresponding more closely to specific strategic problems, should be investigated.

Conclusion. Perhaps the foregoing examples will serve to indicate that the mathematical problems encountered in operations research are not trivial, and that while many important problems can be formulated in precise mathematical form, a precise solution of all but the very simplest of these is beyond the capabilities of mathematics at present. It is to be hoped that more of the work completed during the war can be made available to the mathematical public, and that students can be trained in this and related subjects, so that they may contribute to the peace-time applications of this new field of applied mathematics and so that they may be available to contribute in important ways to our defense in case of war.

Brookhaven National Laboratory 